\title{
MOLECULAR MODELING AND EVALUATION OF THERMAL, CONDUTIMETRIC AND SPECTRUM PROPERTIES OF ACID MALEIC FOR APPLICATION IN PROMISSORY CHEMICAL SYSTEMS
}

\author{
LIMA, Francisco José Santos*; SILVA, Jean Lucas Carvalho da \\ e SILVA, Ademir Oliveira da \\ Universidade Federal do Rio Grande do Norte - Instituto de Química \\ Centro de Ciências Exatas e da Terra, Natal - RN, Brasil \\ ${ }^{*}$ Autor correspondente \\ e-mail:limafjs@yahoo.com
}

Received 12 November 2018; received in revised form 30 November 2018; accepted 14 December 2018

\section{RESUMO}

Neste trabalho foram investigadas as propriedades químicas do ácido maleico $\mathrm{C}_{4} \mathrm{H}_{4} \mathrm{O}_{4}$, através da modelagem e parâmetros de reatividade molecular, medidas de $\mathrm{pH}$ e estudo do equilíbrio ácido-base pelo uso do programa RAİZES 1.0, medidas de condutividade molar, análise térmica, espectros uv-visível e força do oscilador, para avaliar sua potencial viabilidade em formar sistemas químicos complexos promissores, capazes de transformar, via transferência intersistemas, radiações eletromagnéticas dispersas em sistemas emissores de luz seletiva ou conversores de radiação em eletricidade. Foi abservado que o contra-cátion deste ácido possui parâmetros de reatividade moleculares compatíveis para formação de ligações químicas estáveis com espécies metálicas receptoras de elétrons. Seu comportamento eletrolítico 2:1 revela que pode funcionar como quelante versátil, mono- e/ou bidentado, como também mono- e/ou bivalente na formação de com adutos complexos. Sua estabilidade térmica se estende até $100^{\circ} \mathrm{C}$, donde se decompõe quase totalmente até $400^{\circ} \mathrm{C}$ sob atmosfera de $\mathrm{N}_{2}$, finalizando com algum resíduo de carbono. Seus espectros mostram forte absorção na região do ultravioleta, com força do oscilador da ordem dos corantes orgânicos, propriedade que reflete uma investigação criteriosa na área da pesquisa e possível aplicação em sistemas químicos complexos promissores.

Palavras-chave: - ácido maleico, espectroquímica, força do oscilador.

\section{ABSTRACT}

In this work the chemical properties of the maleic acid $\mathrm{C} 4 \mathrm{H} 4 \mathrm{O} 4$ were investigated, through the modeling and molecular reactivity parameters, $\mathrm{pH}$ measurements and acid-base equilibrium study using the RAZES 1.0 program, molar conductivity measurements, thermal analysis, uv-visible spectra and oscillator strength to evaluate their potential viability in forming promising complex chemical systems capable of transforming, via inter-system transfer, dispersed electromagnetic radiation into selective light emitting systems or converters of radiation into electricity. It has been observed that the counter-cation of this acid has compatible molecular reactivity parameters for the formation of stable chemical bonds with metal receptors of electrons. Its 2: 1 electrolytic behavior reveals that it can function as a versatile, mono- and / or bidentate chelator, as well as mono- and / or bivalent chelator in the formation of complex adducts. Its thermal stability extends to $100 \mathrm{oC}$, where it decomposes almost totally to $400 \mathrm{oC}$ under atmosphere of N2, ending with some carbon residue. Their spectra show strong absorption in the ultraviolet region, with the oscillator strength of the order of organic dyes, a property that reflects a careful investigation in the area of research and possible application in promising complex chemical systems.

Keywords: maleic acid, spectrochemistry, oscillator strength. 


\section{INTRODUÇÃO}

O ácido maleico é um sólido branco, de fórmula molecular $\mathrm{C}_{4} \mathrm{H}_{4} \mathrm{O}_{4}$, comercializado na forma de pó, que recentemente tem sido usado na síntese de sistemas complexos, para a verificação das propriedades térmicas, condutimétricas e espectrais. Possui ponto de fusão entre $131-132{ }^{\circ} \mathrm{C}$ e ponto de ebulição 135 ${ }^{\circ} \mathrm{C}$, donde se decompõe. Possui dois hidrogênios ionizáveis com $\mathrm{pKa}_{1}=1,92, \mathrm{pKa}_{2}=6,27 \mathrm{e}$ apresenta solubilidade em água de $78 \mathrm{~g} / 100 \mathrm{~mL}$ a $25{ }^{\circ} \mathrm{C}$. Tem sido largamente utilizado na produção de resinas sintéticas e eletrólitos para capacitores (GREENWOOD, et al, 2006; MELO et al., 2007a; MELO et al., 2007b). A capacidade de seu contra-cátion em formar complexos, mono- e/ou bi-dentados, é um ponto forte para a formação de compostos e aumento de estabilidade de seus complexos em solução e no estado sólido. Suas propriedades físico-químicas apresentam a capacidade de influenciar favoravelmente as características a serem investigadas, e que apresenta interesse aplicativo em sistemas químicos termoestáveis e conversores de radiação.

Este estudo objetiva solucionar situações teóricas e experimentais referentes à síntese de compostos metálicos e a aplicação de técnicas analíticas já consolidadas e inovadas a cada sistema particular. As atividades experimentais deste trabalho justificam seu objetivo que tem como propósito de determiná-las para correlacionar com outros sistemas químicos similares.

\section{METODOLOGIA}

Parâmetros de reatividade teóricos foram obtidos a partir do programa WebLab ViewerPro ${ }^{\circ}$ e da metodologia referenciada (MELO, 2007; WEBLAB, 1998). Os resultados estão mostrados nas Figuras 01 e Tabelas 01 e 02 . Nas práticas experimentais foi utilizado ácido maleico comercial da Cromoline QF de pureza $99 \%$. Medidas de $\mathrm{pH}$ foram realizados em diferentes concentrações em um pHmetro TECNOPON mPA-210 de precisão $\pm 0,005 \mathrm{pH}$ em temperatura ambiente de $25 \pm 1^{\circ} \mathrm{C}$ e foi usado o programa RAÍZES 1.0 elaborado em linguagem QBASIC, para avaliar a concordância teóricoexperimental dos resultados para este propósito (LIMA et al, 2017). As condutividades forram obtidas em um Condutivímetro MS-TECNPON
mCa-150, precisão $\pm 0,001 \mu \mathrm{S} \mathrm{cm}^{-1}$. A análise térmica foi realizada em um equipamento TAInstruments DSC/SDT Q600, a $10{ }^{\circ} \mathrm{C} / \mathrm{min}$ sob atmosfera de $\mathrm{N}_{2}$, da temperatura ambiente até $890^{\circ} \mathrm{C}$. Espectros uv-vis foram registrados em um equipamento UV-VIS SHIMADZU 1650 PC no intervalo de $190-900 \mathrm{~nm}$, com absorbância $\pm 0,002$ e $\lambda \pm 0,1 \mathrm{~nm}$, de precisão, para uma solução aquosa $2,1 \times 10^{-4} \mathrm{~mol} / \mathrm{L}$. Os experimentos foram avaliados em temperatura ambiente de 25 $\pm 1^{\circ} \mathrm{C}$.

\section{RESULTADOS}

Os dados obtidos através de programas de simulações, cálculos e pelos experimentos relatados estão descritos a seguir: A Figura 01 ilustra a modelagem molecular, parâmetros estruturais e eletrônicos do ácido maleico.
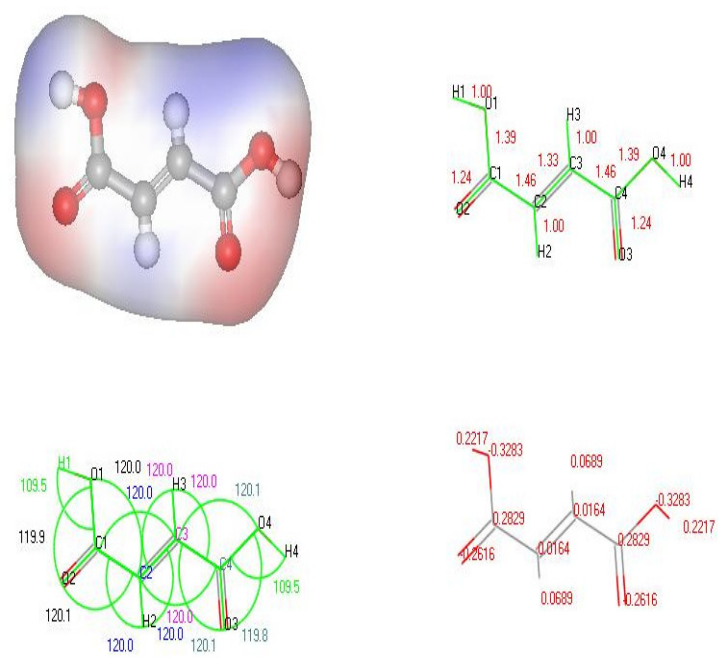

Figura 01 - Modelagem obtida para o ácido maleico através do programa WebLab ViewerPro ${ }^{\circ}$.

Na Tabela 01 estão descritos os valores de carga parcial para cada átomo na molécula neutra e no íon maleato (-1). É possível observar os elementos que apresentam densidades eletrônicas mais prováveis na molécula livre e no íon livre, capazes de promover uma ligação mais efetiva com espécies deficientes de elétrons, ácidos de Lewis, ou íons metálicos para a formação de sistemas químicos complexos promissores. Como esperado as maiores densidades eletrônicas estão mais distribuídas nos elementos mais eletronegativos das espécies. 
Tabela 01 - Cargas parciais para o ácido maleico e o íon maleato $(-1)$.

\begin{tabular}{|c|c|c|}
\hline \multicolumn{3}{|c|}{ Carga Parcial } \\
\hline \multicolumn{2}{|c|}{$\begin{array}{c}\text { Ácido } \\
\text { Maleico }\end{array}$} & Íon Maleato $(-1)$ \\
\hline $\mathrm{H} 1$ & $+0,2217$ & 0 \\
\hline $\mathrm{O} 1$ & $-0,3283$ & $-0,6347$ \\
\hline C1 & $+0,2829$ & $+0,2091$ \\
\hline $\mathrm{O} 2$ & $-0,2616$ & $-0,6347$ \\
\hline C2 & $+0,0164$ & $-0,0045$ \\
\hline $\mathrm{H} 2$ & $+0,0689$ & $+0,0669$ \\
\hline C3 & $+0,0164$ & $+0,0145$ \\
\hline H3 & $+0,0689$ & $+0,0688$ \\
\hline C4 & $+0,2829$ & $+0,2828$ \\
\hline O3 & $-0,2616$ & $-0,2616$ \\
\hline O4 & $-0,3283$ & $-0,3283$ \\
\hline $\mathrm{H} 4$ & $+0,2217$ & $+0,2217$ \\
\hline
\end{tabular}

$\mathrm{Na}$ Tabela 02 estão apresentados os cálculos referentes aos parâmetros de reatividade $\Re$ (LIMA et al., 2007).

Tabela 02 - PRM para o ácido maleico e o íon maleato $(-1)$.

\begin{tabular}{c|c|c|c|c}
\hline \multicolumn{3}{c|}{ Ácido Maleico } & \multicolumn{2}{c}{ Íon Maleato (-1) } \\
\hline & $\Re e$ & $\Re p$ & $\Re e$ & $\Re p$ \\
\hline H1 & - & $+0,1879$ & - & 0 \\
\hline O1 & $-0,2783$ & - & $-0,3405$ & 0 \\
\hline C1 & - & $+0,2398$ & & $+0,2421$ \\
\hline O2 & $-0,2217$ & - & $-0,3405$ & \\
\hline C2 & - & $+0,2398$ & $-0,0024$ & \\
\hline H2 & - & $+0,0584$ & & $+0,3195$ \\
\hline C3 & - & $+0,0139$ & & $+0,3508$ \\
\hline H3 & - & $+0,0584$ & & $+0,0796$ \\
\hline C4 & - & $+0,2398$ & & $+0,3274$ \\
\hline O3 & $-0,2217$ & - & $-0,1404$ & \\
\hline O4 & $-0,2783$ & - & $-0,1762$ & \\
\hline H4 & - & $+0,1879$ & & $+0,2567$ \\
\hline
\end{tabular}

$\mathrm{Na}$ Tabela 03 estão apresentados os valores de $\mathrm{pH}$ experimentais medidos em diferentes concentrações e comparados com valores calculados do equilíbrio ácido-base pelo programa RAİZES 1.0, donde foi utilizado os valores tabelados de pKa ou Ka, para estimar valores teóricos e avaliar a validade dos dados experimentais, conforme metodologia já testada em trabalho anterior (LIMA et al, 2017).

Tabela 03 - Dados calculados pelo uso do programa RAİZES 1.0 e experimentais, para as soluções aquosas do ácido maleico em diferentes concentrações.

\begin{tabular}{l|c|c|c|c}
\hline $\begin{array}{c}\text { Mo } \\
\mathbf{~ m o l} / \mathbf{L}\end{array}$ & $\begin{array}{c}{\left[\mathbf{H}^{+}\right]_{\text {calc }}} \\
\mathbf{~ m o l} / \mathbf{L}\end{array}$ & $\mathbf{p H}_{\text {calc }}$ & $\mathbf{p H}_{\exp }$ & $\begin{array}{c}|\Delta \mathbf{p H}| \\
\text { (cal-exp) }\end{array}$ \\
\hline $1,00 \times 10^{-1}$ & $2,92 \times 10^{-2}$ & 1,54 & 1,81 & 0,27 \\
\hline $1,00 \times 10^{-2}$ & $6,49 \times 10^{-3}$ & 2,19 & 2,57 & 0,38 \\
\hline $2,10 \times 10^{-3}$ & $1,82 \times 10^{-3}$ & 2,74 & 3,02 & 0,28 \\
\hline $1,00 \times 10^{-3}$ & $9,28 \times 10^{-4}$ & 3,03 & 3,25 & 0,22 \\
\hline$\left.{ }^{*}\right) \mathrm{pH}$ da $\mathrm{H}_{2} \mathrm{O}=6,8 ;$ valores experimentais \\
médios medidos em triplicata.
\end{tabular}

A Tabela 04 apresenta valores de $\mathrm{pH}$ e condutividades medidos em temperatura ambiente de $25 \pm 1{ }^{\circ} \mathrm{C}$, obtidos para a solução dos experimentos espectroquímicos. Os valores de condutância molar foram calculados à partir de $\Lambda_{\mathrm{M}}=\left(\mathrm{k}_{\text {sol }}-\mathrm{k}_{\text {solv }}\right) \cdot 10^{3} / \mathrm{M}$, conforme suportado pela literatura pesquisada (GEARY, 1971; GUTTMAN, 1976; COSTA, et al., 2013; LIMA, et al., 2016).

Tabela 04 - Concentração, pH e condutividades do ácido maleico em solução aquosa.

\begin{tabular}{c|c|c|c|c}
\hline & $\begin{array}{c}\mathbf{M} \\
\mathbf{m o l ~ L}^{-1}\end{array}$ & $\mathbf{p H}$ & $\begin{array}{c}\mathbf{k} \\
\mathbf{S ~ c m}^{-1} \\
\mathbf{x} 10^{-6}\end{array}$ & $\begin{array}{c}\Lambda_{\mathbf{M}} \\
\mathbf{S ~ c m}^{2} \\
\mathbf{m o l}^{-1}\end{array}$ \\
\hline $\mathrm{H}_{2} \mathrm{O}$ & 55,33 & 6,8 & 2,52 & $4,55 \times 10^{-5}$ \\
\hline $\mathrm{C}_{4} \mathrm{H}_{4} \mathrm{O}_{4}$ & $\begin{array}{c}2,1 \times 10^{-} \\
3\end{array}$ & 3,02 & 622,7 & 295,3 \\
\hline
\end{tabular}


A Figura 02 mostra a curva de termodecomposição do $\mathrm{C}_{4} \mathrm{H}_{4} \mathrm{O}_{4(\mathrm{~s})}$ em atmosfera de $\mathrm{N}_{2}$.

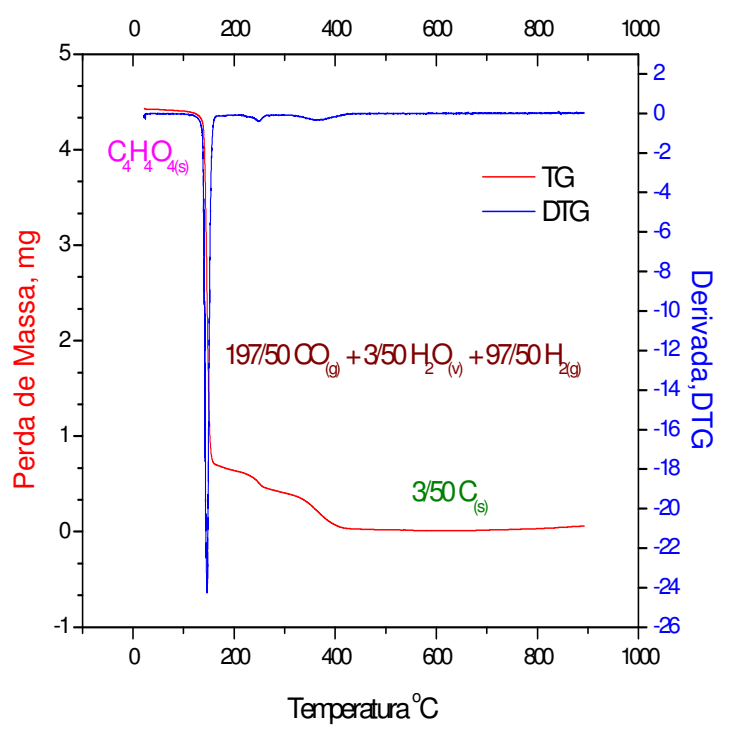

Figura 02 - Curva da termodecomposição do ácido maleico em atmosfera de $\mathrm{N}_{2}$.

$\mathrm{Na}$ Tabela 05 está apresentada uma proposta de atribuição de conversão de reagentes em produtos.

Tabela 05 - Proposta de atribuição para a termodecomposição do ácido maleico em atmosfera de $\mathrm{N}_{2}\left({ }^{*}\right)$.

$\mathrm{C}_{4} \mathrm{H}_{4} \mathrm{O}_{4}(\mathrm{~s}) \rightarrow 197 / 50 \mathrm{CO}_{(\mathrm{g})}+3 / 50 \mathrm{H}_{2} \mathrm{O}_{(\mathrm{v})}+97 / 50 \mathrm{H}_{2(\mathrm{~g})}$ $+3 / 50 \mathrm{C}(\mathrm{s})$

\begin{tabular}{llll}
\hline \multicolumn{2}{l}{ Amostra inicial } & $\begin{array}{l}\text { Produtos } \\
\text { evolados }\end{array}$ & Resíduo \\
\hline Massa & $\begin{array}{l}4,42960 \\
\mathrm{mg}\end{array}$ & $\begin{array}{l}4,40055 \\
\mathrm{mg}\end{array}$ & $0,02905 \mathrm{mg}$ \\
\hline Exp & $100,0 \%$ & $99,34 \%$ & $0,6558 \%$ \\
\hline Teór & $100,0 \%$ & $99,40 \%$ & $0,6207 \%$ \\
\hline$\Delta(\mathrm{T}-\mathrm{E})$ & $0,0 \%$ & $0,06 \%$ & $-0,0351 \%$ \\
\hline
\end{tabular}

$\left({ }^{*}\right)$ Obs. Os percentuais teóricos estão associados a estequiometria proposta que foi atribuída a termodecomposição e os dados estequiométricos foram calculados para a decomposição de $1 \mathrm{~mol}$ de amostra.
A Figura 03 mostra o espectro uv-vis do ácido maleico em solução aquosa $2,1 \times 10^{-4} \mathrm{~mol} / \mathrm{L}$.

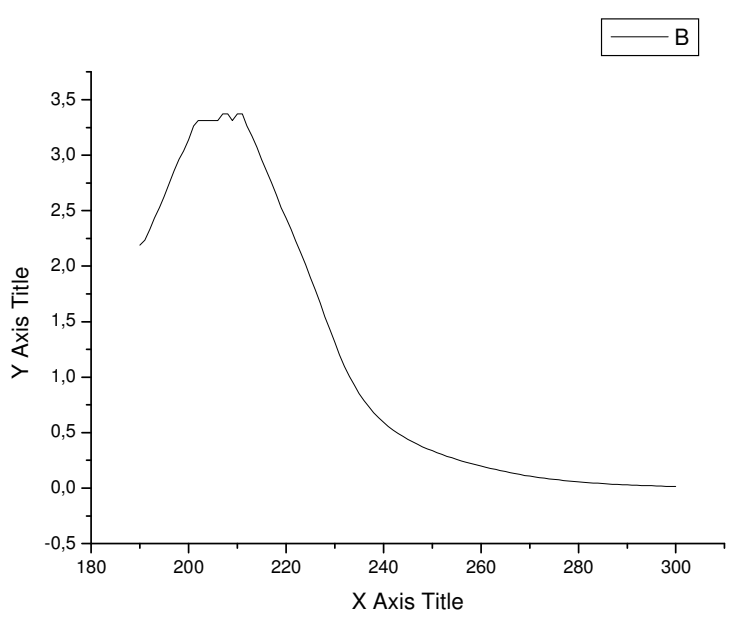

Figura 03 - Espectro uv-vis do ácido maleico

A Tabela 06 contém os dados espectrais obtidos. Utilizamos os métodos citados na literatura para o cálculo da área sob a curva do espectro de absorção e a força do oscilador (DRAGO, 1965; FIGGIS, 1966; LIMA et al., 2014; LIMA et al., 2018)

$$
\text { Drago: } f=4,6 \times 10^{-9} . \int \varepsilon_{(\sigma)} \mathrm{d} \sigma
$$

Figgs: $f=4,32 \times 10^{-9} \cdot \int \varepsilon_{(\sigma)} \mathrm{d} \sigma$

\section{SIMP2FOS}

$$
\begin{gathered}
f=4,319 \times 10^{-9} \cdot\left[9 \eta /\left(\eta^{2}+2\right)^{2}\right] \cdot \int \varepsilon_{(\sigma)} d \sigma \\
\eta=\text { índice de refração }=1
\end{gathered}
$$

Tabela 06 - Parâmetros espectrais do ácido maleico.

\begin{tabular}{c|c|c|c}
\hline Método & $\begin{array}{c}\text { Intervalo } \\
(\mathbf{n m})\end{array}$ & $\begin{array}{c}\text { Área } \\
\left(\mathbf{c m}^{-1} \mathbf{)}\right. \\
\mathbf{X 1 0 ^ { 4 }}\end{array}$ & $\begin{array}{c}\text { Força do } \\
\text { Oscilador }\end{array}$ \\
\hline $\begin{array}{c}\text { Gaussiana } \\
\text { Drago }\end{array}$ & $190-227$ & 2,894 & 0,6339 \\
\hline $\begin{array}{c}\text { Gaussiana } \\
\text { Figgs }\end{array}$ & $190-227$ & 2,894 & 0.5953 \\
\hline SIMP2FOS & $190-300$ & 2,454 & 0,5049 \\
\hline
\end{tabular}




\section{DISCUSSÃO E CONCLUSÕES}

Os parâmetros de reatividade para a molécula isolada, mostraram que, no ânion monovalente em relação ao ácido, há um aumento de $93 \%$ em termos da carga parcial e $22 \%$ no valor de Re sobre o O1 da hidroxila $\mathrm{OH}$, para o primeiro hidrogênio ionizável, o que mostra sua viabilidade como contra-cátion monovalente, na formação de sistemas complexos com espécies metálicas, ácidos de Lewis ou receptores de elétrons. Em relação ao $\mathrm{O} 2$ ligado na carbonila $\mathrm{C}=\mathrm{O}$, o aumento relativo é maior ainda, em torno de $142 \%$ em termos de carga parcial e $54 \%$ em termos de Re, o que faz do grupo [O=CR-O-] no íon molecular $\mathrm{C}_{4} \mathrm{H}_{3} \mathrm{O}_{4}{ }^{-}$, uma espécie doadora de elétrons de grande relevância para esta aplicação, podendo formar espécies mono- e bidentadas, assim como mono- e bivalentes pela perda de mais um hidrogênio ionizável.

O programa RAİZES 1.0, elaborado em linguagem QBASIC, com o propósito de ser empregado como metodologia para averiguar a validade das medidas experimentais de $\mathrm{pH}$ obtidas em laboratório para diferentes sistemas, como também avaliar a consistência do equilíbrio ácido-base das soluções aquosas do ácido maleico, que revelou boa concordância entre os dados calculados e experimentais, como descrito na Tabela 03 (LIMA et al, 2017).

A condutância molar revelou que o ácido maleico é um eletrólito de comportamento 2:1 em solução aquosa, com condutância molar $\Lambda_{\mathrm{M}}=$ 295,3 S cm ${ }^{2} \mathrm{~mol}^{-1}$, segundo 0 estudo comparativo suportado por ampla literatura (GEARY, 1971; GUTTMAN, 1976; THOMPSON, 1979; HART, 1987; MELO, et al., 2009; COSTA et al., 2013).

O comportamento térmico sob atmosfera de $\mathrm{N}_{2}$, apresentou termoestabilidade até $100^{\circ} \mathrm{C}$, donde se inicia as primeiras perdas de massa, com uma decomposição térmica completa idealizada como;

$$
\begin{gathered}
\mathrm{C}_{4} \mathrm{H}_{4} \mathrm{O}_{4(\mathrm{~s})} \rightarrow \underset{(\mathrm{CO})}{2 \mathrm{CO}_{(\mathrm{g})}}+2 \mathrm{H}_{2} \mathrm{O}_{(\mathrm{g})}+2 \mathrm{C}, \\
\text { ou } \\
\mathrm{C}_{4} \mathrm{H}_{4} \mathrm{O}_{4(\mathrm{~s})} \rightarrow 4 \mathrm{CO}_{(\mathrm{g})}+2 \mathrm{H}_{2(\mathrm{~g}),}
\end{gathered}
$$

pela ausência de oxigênio suficiente no composto, para a total oxidação da substância.
$\mathrm{Na}$ prática foi observado que a decomposição ocorreu em três etapas visíveis, sendo atribuída neste trabalho e compatíveis teoricamente com o processo global,

$$
\begin{gathered}
\mathrm{C}_{4} \mathrm{H}_{4} \mathrm{O}_{4(\mathrm{~s})} \rightarrow 197 / 50 \mathrm{CO}_{(\mathrm{g})}+3 / 50 \mathrm{H}_{2} \mathrm{O}_{(\mathrm{v})}+ \\
97 / 50 \mathrm{H}_{2(\mathrm{~g})}+3 / 50 \mathrm{C}_{(\mathrm{s})}, \\
\text { ou, } \\
\mathrm{C}_{4} \mathrm{H}_{4} \mathrm{O}_{4(\mathrm{~s})} \rightarrow 3,94 \mathrm{CO}_{(\mathrm{g})}+0,06 \mathrm{H}_{2} \mathrm{O}_{(\mathrm{v})}+ \\
1,94 \mathrm{H}_{2(\mathrm{~g})}+0,06 \mathrm{C}_{(\mathrm{s})}, \\
\text { ou, } \\
50 \mathrm{C}_{4} \mathrm{H}_{4} \mathrm{O}_{4(\mathrm{~s})} \rightarrow 197 \mathrm{CO}_{(\mathrm{g})}+3 \mathrm{H}_{2} \mathrm{O}_{(\mathrm{v})}+ \\
97 \mathrm{H}_{2(\mathrm{~g})}+3 \mathrm{C}_{(\mathrm{s})},
\end{gathered}
$$

mostrado na Tabela 05, finalizando com um resíduo carbonífero, obtido a uma temperatura final de $436,1^{\circ} \mathrm{C}$, que pode ser associado ao negro de fumo, um subproduto amorfo obtido da queima incompleta de compostos orgânicos, provavelmente não estequiométrico, polimérico, como também, possuir frações cristalinas de fulerenos, conhecidos como estruturas buckminster (LEE., 2000).

O espectro eletrônico (Figura 03) indica altas intensidades para as transições moleculares registradas, com valor de absorbância máxima de $A_{\max }=3,3737 \mathrm{e}$ absortividade molar $\varepsilon_{\max }=16065 \mathrm{~L} \mathrm{~mol}^{-1} \mathrm{~cm}^{-1}$, que ocorrem apenas na região do uv com forte absorção, sendo a transição principal, atribuída a um mecanismo permitido por dipolo elétrico do tipo $\mathrm{n} \rightarrow \pi^{*}$ (DRAGO, 1965; SILVERSTEIN, et al., 1994; LIMA, et al., 1996; PAVIA, et al., 2010), revelando valores elevados para a força do oscilador, da ordem dos corantes orgânicos de aproximadamente 1 (KAUZMANN, 1957; PITZER, 1965; ADAMSON, 1986; LIMA, et al., 1996), mostrados na Tabela 06. Analisando os valores obtidos para a força do oscilados pelos métodos descritos para pré-avaliação e comparação, observa-se que são próximos, sendo o mais preciso o método elaborado pela "Regra de Simpson" que avalia mais criteriosamente a área sob curvas irregulares, e que foi realizado através do programa SIMP2FOS (LIMA, et al., 1996).

Apesar de o ácido maleico possuir espécies incolores em solução aquosa, estas absorvem fortemente na região do ultravioleta, o 
que pode ser potencialmente aplicado em conversão radiativa por emissão de luz, através da transferência de energia inter-sistemas com outras espécies receptoras intramoleculares, usados na aplicação tecnológica em dispositivos eletro-eletrônicos.

\section{AGRADECIMENTOS}

A UFRN/PROPESQ/CNPq, pelo incentivo à pesquisa fundamental e ao $I Q$ pelas análises térmicas e espectros uv-vis.

Este trabalho foi desenvolvido com o apoio do Projeto de Pesquisa PVB 11090-2014 Síntese, Caracterização e Estudos Espectroquímicos de Maleatos de Lantanídeos com Aminóxidos Aromáticos, cadastrado na PróReitoria de Pesquisa da UFRN.

\section{REFERÊNCIAS}

1. ADAMSON, A. W. 1986 - Physical Chemistry, Academic Press, Inc., Florida.

2. COSTA, L. H. M., LIMA, F. J. S. e SILVA, A. O., 2013 - Síntese, Complexometria, Análise Térmica e Condutância Molar dos Cloretos de Lantânio, Neodímio e Érbio Hidratados Periódico TchêQuímica, 10(19), 38-45.

3. DRAGO, R. S., 1965 - Physical Methods in Inorganic Chemistry -, Vau Nostrand Reinhold Company, printed Holland.

4. FIGGIS, B. N., Introduction To Ligands Fields, 1966 - Interscience Publishers,

5. GEARY, W. J., 1971 - The Use Of Conductivity Measurements In Organic Solvents For The Characterization Of Coordination Compounds - Coord. Chem. Rev., 81-122.

6. GREENWOOD N, EARNSHAW A, 2006 Chemistry of the Elements 2nd ed. Oxford: Butterworth-Heinemann.

7. GUTTMAN, V., 1976 - Empirical Parameters for Donor and Acceptor Properties of Solvents - Eletrochim. Acta. 21, 661-70.

8. HART, F. A.,1987 - Scandium, Yttrium and The Lanthanides - Comprehensive Coordination Chemistry - Ed. Sir Geoffrey Wilkinson, F. R. S. - 1a Ed., Vol. 3 - Main Group Early Transition Elements, Ch. 39, 1059-1127, Pergamon Press, 1a Ed., Oxford, England.
9. KAUZMANN, W. 1957 - Quantum Chemistry - Academic Press, Inc., New York.

10. LEE, J. D., 2000 - Quimica Inorganica Não Tao Concisa. Ed. Edgard Blucher LTDA, SP

11. LIMA, F. J. S., BRITO, H. F., SILVA, A. G., SILVA, A. O., BRAGA, C. C. M., LIMA, A. J. P., CARDOSO, M. C. C., 1996 - O Uso da Força do Oscilador na Avaliação de Intensidades Espectrais - Anais da Associação Brasileira de Química, 45 (1), 31-35.

12. LIMA, F. J. S.; MELO, R. M.; SILVA, A. O. e BRAGA, C. C. M., 2007 - Parâmetros de Reatividade Molecular - Periódico Tchêquímica, 4(7), 7-15.

13. LIMA, F. J. S. COSTA, L. H. M e SILVA, A. O., 2014 - Estudos Espectroquímicos do Íon $\mathrm{UO}_{2}{ }^{2+}$ Coordenado no Acetato de Uranila Periódico Tchêquímica, 11(22), 33-46.

14. LIMA, F. J. S, COSTA, L. H. M., AZEVEDO, D. M., SILVA, A. O., e CLAUDECE, F. P., 2016 - Parâmetros de Reatividade Molecular e a Correlação Com a Condutividade Molar da Quinolina-N-Óxido, Nicotinamida-N-Óxido e a 2,2Dithiobispiridina-N-Óxido , Periódico Tchêquímica, 13(26), 88-96.

15. LIMA, S. G. M., LIMA, F. J. S., PEREIRA, F. C. e CRUZ, T. J. T., 2017 - Modelagem Molecular, Medidas Condutimétricas e Espectros Uv-Vis do Ácido Ascórbico Para Formação de Sistemas Químicos Complexos - Trabalho apresentado na CIENTEC - XXIII SEMANA DE CIÊNCIA, TECNOLOGIA E CULTURA, de 25 a 27 de outubro em Natal- RN.

16. LIMA, F. J. S., COSTA, L. H. M. da, SILVA, A. O. da, PEREIRA, F. C., 2018 Espectroscopia de Absorção Molecular na Região do Uv-Vis Para os Ligantes Quinolina-N-Óxido, Nicotinamida-N-Óxido e 2,2-Dithiobispiridina-N-Óxido - Periódico Tchêquímica, 15(29), 164-170.

17. MELO, R. G. P.; MARTINS, C. Q. S.; MELO, R. M.; SILVA., A. L. C.; LIMA, F. J. S. e BRAGA., C. C. M, 2007a - Avaliação de Parâmetros de Reatividade Molecular Para os Isômeros Ácido Maleico e Fumárico, XLVII Congresso Brasileiro de Química $A B Q / R N, 17$ a 21 de setembro.

18. MELO, R. M., LIMA, F. J. S., e SILVA, A. O., 
2007b - Estudo Térmico e Modelagem Molecular dos Maleatos de Lantanídeos Hidratados, XVIII Congresso de Iniciação Científica da UFRN - CIC, 02 a 05 de outubro.

19. MELO, R. M., LIMA, F. J. S., SILVA, A. O. e BRAGA, C. C. M., 2009 - Sintese e Caracterizacao dos Maleatos de Lantanio, Neodimio e Erbio. - Periodico Tche-Quimica, 06, 11, 31-42.

20. PAVIA, D. L.; LAMPMAN, G. M.; KRIZ, G. S. e VYVYAN, J. R.; 2010 - Introdução à Espectroscopia; São Paulo: 4. Ed. Norteamericana.

21. PITZER, K. S., 1965 - Quantum Chemistry Prentice-Hall, Inc. 7a Ed.

22. SILVERSTEIN, R. M., BASSLLER, G. C. and MORRILL, T. C. 1994, Identificação Espectrométrica de Compostos Orgânicos, 5a. Ed. Guanabara Koogan S.A

23. THOMPSON, L. C. 1979 - Complexes Handbook on the Physics and Chemistry of Rare Earths, V.3, Ch. 25, 209-97.

24. WEBLAB VIEWERPRO, Copyright (C) 1998, Molecular Simulations, Inc., Web Lab and ViewerPro are trademarks of Molecular Simulation Inc 